\title{
Why choose high volume online post-dilution hemodiafiltration?
}

Concise title: High volume online post-dilution hemodiafiltration

Carlo Basile $^{1}$, Andrew Davenport ${ }^{2}$, Peter J Blankestijn ${ }^{3}$

${ }^{1}$ Clinical Research Branch, Division of Nephrology, Miulli General Hospital, Acquaviva delle Fonti, Italy, ${ }^{2}$ University College London, Centre for Nephrology, Royal Free Hospital, University College London Medical School, London, UK, ${ }^{3}$ Department of Nephrology, University Medical Center Utrecht, Utrecht, The Netherlands

Compliance with ethical standards

The authors declare that they have no conflict of interest.

No funding agency granted the present study.

Corresponding author:

Carlo Basile, MD

Via C. Battisti 192

74121 Taranto

Tel + 39-099-4773688

Fax $+39-080-762165$

E-mail: basile.miulli@ libero.it 


\section{Why do we need new dialysis treatment options?}

Hemodialysis (HD) is the standard renal replacement therapy for patients with end-stage kidney disease (ESKD), with more than 2 million patients now treated worldwide. Although significant advances are being made in the understanding of uremic toxins and despite major technological improvements over the last decades, morbidity and mortality rates remain unacceptable high. According to the 2013 US Renal Data System Annual data Report, adjusted rates of all-cause mortality are 6.5 - 7.9 times greater for dialysis patients than for age and gender matched individuals from the general population (http://www.usrds.org/atlas.aspx). In Europe, the 2011 Annual Report from the European Renal Association - European Dialysis and Transplant Association Registry (1), shows a two-year survival probability for incident dialysis patients of only $71.6 \%$. Five-year survival in UK is just marginally above that for ovarian cancer, but markedly less than that for colon cancer, and much lower than that for acute myocardial infarction or stroke (2). Therefore, there is an unmet and urgent medical need to improve the prognosis for ESKD patients.

\section{Why do we need convective clearance?}

Standard HD with low-permeable membranes has been shown to be an effective treatment for removing low-molecular weight solutes, such as urea by diffusion, but simply increasing urea clearance did not improve survival (3). Low permeable dialyzers are unable to effecively clear larger molecular weight azotemic toxins and protein-bound toxins. There is increasing evidence from cell culture studies, small animal models and clinical observational studies that these compounds cause endothelial and cardiac damage and are associated with increased cardiovascular mortality (4 - 6). Successful removal (7 - 10) of these larger solutes and proteinbound compounds by hemodiafiltration (HDF) may therefore offer a viable approach to improve dialysis patient survival. 
In the last few years, three large, prospective, randomized controlled trials (RCTs) have been conducted in different European countries to compare survival outcomes in prevalent patients receveing conventional HD and online post-dilution HDF (OL-HDF) (11 - 13).

In the Convective Transport Study (CONTRAST) (11), 714 prevalent HD patients were randomly assigned to undergo either OL-HDF (post-dilution, target convection volume 6 liters/h) or lowflux HD. The primary outcome was all-cause mortality. The main secondary endpoint was a composite of fatal and non-fatal major cardiovascular events. After a mean follow-up of 3.0 years, the incidence of all-cause mortality was not affected by treatment assignment. However, subgroup analysis suggests benefit on all-cause mortality (hazard ratio 0.57 ; $<0.016$ ) among those patients treated with high convection volumes (> 20 liters/treatment)

The Turkish OL-HDF Study (12) compared the effects on morbidity and mortality outcomes for OL-HDF and high-flux HD. Seven hundred and eighty-two patients were randomly assigned at a 1:1 ratio to either OL-HDF or high-flux HD. The follow-up period was 2 years. The primary outcome was the composite of death from any cause and non-fatal cardiovascular events. There was no statistically significantly difference between the two treatments. However, in a post-hoc analysis treatment with OL-HDF achieving a substitution volume $>17.4$ liters was associated with a $46 \%$ reduction ratio for overall mortality $(\mathrm{p}=0.02)$ and a $71 \%$ reduction ratio for cardiovascular mortality $(\mathrm{p}=0.003)$.

Finally, in Catalonia (Spain), the ESHOL trial (13) assigned 906 patients either to continue HD or to switch to high-efficiency OL-HDF. ESHOL is the first randomized study to show a significant advantage for OL-HDF, reducing all-cause mortality by $30 \%$ (primary outcome, $\mathrm{p}=0.01$ ), cardiovascular mortality by $33 \%$ (secondary outcome, $\mathrm{p}=0.06$ ) and infection-related mortality by $55 \%$ (secondary outcome, $\mathrm{p}=0.03$ ). Mean convective volumes were 23.7 liters/session; mean blood flow rate $387 \mathrm{ml} / \mathrm{min}$ and mean dialysis time $236 \mathrm{~min}$.

Four methods summarizing data from epidemiological studies are currently used: type I: qualitative summary, the narrative review article; type II: quantitative summary of published data 
(usually called meta-analysis); type III: re-analysis of individual data based on primary studies (usually called pooled analysis); type IV: prospectively planned, pooled analysis of several studies, where pooling is already a part of the protocol (14). A meta-analysis is considered the highest evidence level, yet large meta-analyses on convective therapies have been published in the last two years, which, however, showed a discordant outcome (15 - 18). It appears that these analyses differ in the number of studies and patients included, the definitions of comparator and intervention therapy, and types of studies, varying from small observational to large prospective RCTs. In one meta-analysis low-flux HD was the reference therapy (17), while both low-flux and high-flux HD were reference therapies in three others $(15,16,18)$. Considering the intervention arm, inclusion covered different combinations of high-flux HD, off-line HDF, hemofiltration, acetate-free biofiltration and post-dilution OL-HDF. Nistor et al.compared HD to HDF and acetate-free biofiltration (16), Susantitaphong et al. analyzed low-flux HD in relation to high-flux HD and HDF (17), and Mostovaya et al considered only RCTs in which OL-HDF was compared to HD (15). Obviously, conclusions depend not only on data extraction and estimation of risk of bias, but also on the definition of convective therapies applied. In the mortality meta-analysis by Nistor et al. both an acetate-free biofiltration study and an off-line HDF study were included (16). However, these modalities can hardly be considered modern convective therapies, as the convection volumes of $10-12$ liters/session achieved are similar to the amount of internal filtration obtained with high-flux HD and completely different from high volume HDF. After removing the two studies from the meta-analysis by Nistor et al. (16), the relative risk of mortality becomes 0.82 (95\% confidence interval $0.72-0.93$ ), which appears to be a better risk estimate (15). A revised definition of HDF was published by the EuDial working group, in which HDF is a blood purification therapy combining diffusive and convective solute transport using a high-flux membrane characterized by an ultrafiltration coefficient $>20 \mathrm{ml} / / \mathrm{h} / \mathrm{mmHg} / \mathrm{m}^{2}$ and a sieving coefficient for $\beta_{2}$ microglobulin $>0.6$; convective transport is achieved by an effective convection volume of at least $20 \%$ of the total blood volume processed (19). In our opinion, a statement on 
today's convective therapies should be based on a convection volume of at least $17-23$ liters/session in the online post-dilution mode. The only meta-analysis that largely fullfils this criterion reported that HDF was associated with a decreased risk for all cause and cardiovascular mortality of $16 \%$ and $27 \%$, respectively (15).

Some of the problems that arise with type II meta-analysis are avoidable if individual data from all studies are available. Publication bias may be reduced in comparison to Type I and II metaanalysis as even unpublished data can be included. With individual data, statistical re-analysis can be performed. This includes some inclusion criteria for all studies, a unified definition of variables, and new statistical modelling (14). The three RCTs (11 - 13) contain several potential risks of bias, leading to either an over- or underestimation of the true effect. An individual participant data meta-analytic approach might solve several methodological problems and increase the level of evidence for a potential effect of HDF on morbidity and mortality. In fact, this analysis was performed using data from CONTRAST, Turkish OL-HDF Study, ESHOL and a fourth as yet not published French $\operatorname{HDF}$ study $(11-13,20)$. The analysis was intentionally restricted to the above studies for two main reasons. First, to ensure high quality of the event adjudication, reasonable power for mortality endpoints and low risk of bias, "studies had to be designed to examine the effect of online HDF on mortality endpoints" (21). Secondly, not all convective therapies are equal (22); therefore, only RCTs comparing online HDF with HD were considered. This study showed that online HDF reduced the risk of all-cause mortality by $14 \%$ and cardiovascular mortality by $23 \%$. The largest survival benefit was for patients receiving the highest delivered convection volume (> 23 liters per $1.73 \mathrm{~m}^{2}$ body surface area per session), with a multivariable adjusted hazard ratio of 0.78 for all-cause mortality and 0.69 for cardiovascular disease mortality (21). A further analysis of the same data tested different methods to standardize OL-HDF convection volume on all-cause and cardiovascular mortality compared to HD. Allcause mortality was reduced when the convective dose was unstandardized or standardized to body surface area and total body water. Standardization by body weight or body mass index gave 
no significant survival advantage (23). The issue of optimal convection volume for improving patient outcomes was recently studied in an international incident dialysis cohort treated with OLHDF (24). In total, 2293 patients with a minimum of 2 years of follow-up were analyzed using advanced statistical tools, including cubic spline analyses for determination of convection volume range over which a survival increase was observed. The relative survival rate of OL-HDF patients, adjusted for age, gender, Charlson comorbidity index, vascular access, albumin, C-reactive protein, and dialysis dose, was found to increase at about 55 liters/week of convection volume and to stay increased up to about 75 liters/week. The relative survival gain is minimal above a convection dose of over 75 liters/week, meaning that no additional outcome benefit could be expected at such very high convection volumes (24). Few studies have examined the association between delivered convection volume and mortality outcomes. The prospective cohort Dialysis Outcome Practice Patterns Sudy (DOPPS) reported that the mortality risk was 35\% lower in European ESKD patients receiving a minimum of 15 liters/session of replacement fluid (equalling $\sim 17$ liters of convection volume) than in the control population receiving standard HD, even after adjustment for age, comorbidities, urea clearance and local practice patterns (7). A second prospective observational study of more than 700 ESKD patients over a 3-year period also suggested that high volume HDF was associated with lower mortality risk (10). However, a retrospective analysis of the entire Romanian dialysed population from the Fresenius Medical Care Database did not show any association between convective volume (the basal median convective volume was 22.2 liters/session) and mortality risk (25). This study suggested that HDF treatment could reduce all-cause mortality in incident and prevalent patients even after correction for different confounders. Interestingly, an additional survival benefit could be observed in incident patients (25). This issue remains still to be fully settled, since so far there are no large RCTs comparing HDF with HD in incident patients.

As almost all patients enrolled into the the pooled individual participant data analysis $(21,23)$ were treated in a thrice-weekly schedule, this dose equates to $\geq 69$ liters/week. One possible 
criticism is that this dose and improved survival is confounded by selection bias, i.e. that high dose is only achievable in healthy patients with good functioning vascular access, with low mortality risk. "While extensive statistical adjustments did not materially alter the main findings, residual confounding may always remain" (21). Furthermore, a major limitation of the study is that "the included trials were not designed to assess the impact of delivered convection volume with OL-HDF and mortality outcomes. The analyses on convection values should therefore be interpreted as an observational analysis in which residual confounding cannot be excluded. A new RCT targeting different convection volumes would be needed to definitively examine the doseresponse effect shown in this study" (21). Finally, the analysis was better powered than the individual trials to study whether, compared with HD, the effect of OL-HDF differed across certain patient populations. In this sufficiently powered analysis, free from selection bias due to informative censoring, HDF was found to beneficially improve survival and reduce cardiovascular mortality consistently across a variety of important clinical patient subgroups (21).

A meta analysis is considered the highest evidence level, To further answer the question whether HD treatment with convective therapies improves clinical outcomes, large meta analyses on convective therapies have been published in the last two years, which, however, showed at discordant outeome (19-22). It appears that these analyses differ in the number of studies and patients included, the definitions of comparator and intervention therapy, and types of studies, varying from small observational to large prospective RCTs. In one meta-analysis low flux HD was the reference therapy (21), while both low flux and high flux HD were reference therapies in three others $(19,20,22)$. Considering the intervention arm, inclusion covered different combinations of high flux HD, off line HDF, hemofiltration, acetate free biofiltration and postdilution OL HDF. Nistor et al.compared HD to HDF and acetate free biofiltration (20), Susantitapheng et al. analyzed low flux HD in relation to high flux HD and HDF (21), and Mostovaya et al considered only RCTs in which OL-HDF was compared to HD (19). Obviously, conclusions depend not only on data extraction and estimation of risk of bias, but also on the 
definition of convective therapies applied. In the mortality meta analysis by Nistor et al. both an acetate free biofiltration study and an off line HDF study were included (20). However, these modalities can hardly Some of these modalities, however, cannot really be considered modern convective therapies, as the convection volumes of $10 \quad 12$ liters/session achieved are similar to the amount of internal filtration obtained with high-flux HD and completely different from high volume HDF. After removing the two studies from the meta-analysis by Nistor et al. (20), the relative risk of mortality becomes 0.82 (95\% confidence interval $0.72 \quad 0.93$ ), which appears to be a better risk estimate (15). A revised definition of HDF was published by the EuDial working group (30), in which HDF is a blood purification therapy combining diffusive and convective solute transport using a high flux membrane characterized by an ultrafiltration coefficient $>20$ $\mathrm{m} / \mathrm{h} / \mathrm{mmmHg} / \mathrm{m}^{2}$ and a sieving coefficient for $\beta_{2}-$ mieroglobulin $\rightarrow 0.6$; convective transport is achieved by an effective convection volume of at least $20 \%$ of the total blood volume processed. In our opinion, a statement on today's convective therapies should be based on a convection volume of at least $17-23$ liters/session in the online post dilution mode. The only meta analysis that largely fullfils this criterion reported that HDF was associated with a decreased risk for all eause and cardiovascular mortality of $16 \%$ and $27 \%$, respectively (19).

Taken together, the above studies $(11-13,15,21,23)$ support the conclusion that high volume post-dilution OL-HDF is associated with improved overall survival. This advantage results predominantly from a lower cardiovascular mortality.

\section{Why should high volume post-dilution OL-HDF be associated with improved overall survival?}

This crucial question generates at least two important questions: first, are the aforementioned RCTs $(11$ - 13) confounded by the favorable profile of HDF patients at entry to the study? Although this cannot be completely ruled out, it should be mentioned that extensive corrections were made in all RCTs. Secondly, what could be the underlying mechanisms? As already stated, 
the survival advantage results predominantly from lower cardiovascular mortality. According to the 2015 US Renal Data System Annual data Report, cardiovascular mortality of US ESKD patients in 2013 accounted for $53.1 \%$ of deaths: the main causes were arrhythmia/cardiac arrest (37.0\%), acute myocardial infarction and atherosclerotic heart diseases (6.7\%), congestive heart failure (5.8\%) and cerebrovascular accidents (3.1\%) (http://www.usrds.org/atlas.aspx). In theory, both a decrease in the incidence of heart failure, ischemic heart disease, sudden cardiac death and stroke may play a role. Interestingly, in ESHOL (13) a reduction in stroke was found, while the incidence of heart failure and ischemic heart disease was similar. None of the three recent published RCTs (11 - 13) found a difference in sudden cardiac death between HD and HDF. Echocardiographic studies reported in the literature indicate that the functional and structural deterioration of the left ventricle over time in HD patients could be mitigated or even prevented in HDF: analysis of a large subset of the CONTRAST cohort (11) revealed that, whereas both left ventricle mass and ejection fraction deteriorated over time in the HD patients, these parameters remained stable in the HDF group (26). Moreover, a recent RCT reported that high volume HDF (> 22 liters/session) prevented the endothelial dysfunction and stiffening of conduit arteries that was observed in HD patients (27). Thus, the advantage of high volume post-dilution OL-HDF could be possibly due to better preservation of left ventricle mass and function.

Improved stability of intra-dialytic blood pressure could also potentially contribute to the beneficial effect of high volume OL-HDF on survival. Intra-dialytic hypotension (IHD) is a common problem during $\mathrm{HD}$, and has been related to cardiac stunning, bowel ischemia and brain hypoperfusion. The occurrence of IDH was not documented in the primary CONTRAST paper (11); it was a secondary outcome in the Turkish study (12), but results were not described in their primary paper; in the ESHOL trial (13) IDH was monitored and the results were reported: in the HDF group, there were 679 episodes of IDH per 100 patient-years $v s .938$ episodes of IDH with HD, a difference which was highly statistically significant. Observational studies now suggest that IDH is strongly associated with poor outcomes, and especially poor cardiovascular outcomes. 
Interestingly, treatment with cooled dialysate reduces IDH, HD-induced brain injury (28) and improves cardiovascular survival (29). Two large RCTs reported that blood pressure stability during HDF was superior to HD $(13,30)$, but not but not a third one (12). However, when cooled dialysate was used both in HDF and HD, hemodynamic changes, as measured as blood pressure, blood volume, cardiac output and microcirculation, did not differ (31). Hence, it appears that intradialytic hemodynamic stability is better preserved during HDF than during standard HD, but analogous to cooled-dialysate HD. Unfortunalely, none of the three recent RCTs (11 - 13) on high volume OL-HDF reported dialysate temperature. Temperature balance studies would suggest that if the cause of slight extracorporeal cooling with post-dilution HDF is due to the slightly lower temperature of the substitution fluid stream, then all other things being equal, increasing the substitution fluid infusion flow rate should result in more extracorporeal circuit cooling (32).

There is no compelling evidence to-date that high volume post-dilution OL-HDF reduces mortality by improvements in traditional (such as high blood pressure and cholesterol levels) and non-traditional risk factors (such as reducing azotemic toxins or reducing the bio-incompatibility of the extra-corporeal system). In terms of solute removal, neither $\mathrm{Kt} / \mathrm{V}_{\text {urea }}$ nor $\beta_{2}$ microglobulin were associated with survival. None of the meta-analyses or RCTs reported a decline in other causes of death, such as withdrawal from dialysis or malignancies. Infectious complications are usually linked to bacterial spread from vascular access, particularly in the case of central venous catheters, and respiratory infections. Whereas no overall difference was observed in CONTRAST (11), infection-related mortality in ESHOL (13) was lowest in HDF patients. Whether this reduction in infection resulted from a lower central venous catheter usage in the HDF group (7\% in the HDF group vs. HD 13\%) or from the high convection volumes applied remains to be established.

\section{Why choose high volume post-dilution OL-HDF?}

Significant advances have been made in the understanding of uremic toxins and major 
technological improvements have been achieved over the last decades. High volume post-dilution OL-HDF appears to be one of the most important technological improvements of the last decade. A revised definition of HDF was published by the EuDial working group (30), in which HDF is a blood purification therapy combining diffusive and convective solute transport using a high flux membrane characterized by an ultrafiltration coefficient $>20 \mathrm{ml} / \mathrm{h} / \mathrm{mmmHg} / \mathrm{m}^{2}$ and a sieving coefficient for $\beta_{z}$-microglobulin $>0.6$; convective transport is achieved by an effective convection volume of at least $20 \%$ of the total blood volume processed. Recommendations to achieve the optimal high volume post-dilution OL-HDF convection dose are reported in Table 1.

Why choose high volume post-dilution OL-HDF? Because it is associated with improved overall survival. This advantage results predominantly from reduced cardiovascular mortality, possibly due to better preservation of left ventricle mass and function. Improved intra-dialytic blood pressure stability may additionally contribute to the beneficial effect of high volume post-dilution OL-HDF on survival. The improved patient survival associated with high volume post-dilution OL-HDF is not restricted to selected patient groups, depending upon age, comorbidity or dialysis vintage. There is no compelling evidence to-date that high volume post-dilution OL-HDF reduces mortality by improvements in traditional and non-traditional cardiovascular risk factors. There are still no studies nor case reports published describing adverse clinical outcomes in more than 20 years of HDF clinical experience. 


\section{REFERENCES}

1. ERA-EDTA Registry: ERA-EDTA Registry Annual Report 2011. Academic Medical Center, Department of Medical Informatics, Amsterdam, The Netherlands, 2013

2. Udayaraj U, Pruthi R, Casula A et al (2013). UK Renal Registry $16^{\text {th }}$ annual report: chapter 6 demographics and outcomes of patients from different ethnic groups on renal replacement therapy in the UK. Nephron Clin Pract 125: 111 - 125

3. Eknoyan G, Beck GJ, Cheung AK et al. Effect of dialysate dose and membrane flux in maintenance hemodialysis (2002). N Engl J Med 347: 2010 - 2019

4. Vanholder R, Van Laecke S, Glorieux G (2008). What is new in uremic toxicity? Pediatr Nephrol 23: $1211-1221$

5. Krieter DH, Hackl A, Rodriguez A et al (2010). Protein-bound uraemic toxin removal in haemodialysis and post-dilution haemodiafiltration. Nephrol Dial Transplant 25: 212 - 218

6. Basile C, Libutti P, Di Turo AL et al (2011). Removal of uraemic retention solutes in standard bicarbonate haemodialysis and long-hour slow-flow bicarbonate haemodialysis. Nephrol Dial Transplant 26: 1296 - 1303

7. Canaud B, Bragg-Gresham JL, Marshall MR et al (2006). Mortality risk for patients receiving hemodiafiltration versus hemodialysis: European results from the DOPPS. Kidney Int 69: 2087 2093

8. Bosch JP, Lew SQ, Barlee V et al (2006). Clinical use of high-efficiency hemodialysis treatments: long-term assessment. Hemodial Int 10: 73 - 81

9. Vilar E, Fry AC, Wellsted D et al (2009). Long-term outcomes in online hemodiafiltration and high-flux hemodialysis: a comparative analysis. Clin J Am Soc Nephrol 4: 1944 - 1953

10. Panichi V, Rizza GM, Paoletti S et al (2008). Chronic inflammation and mortality in haemodialysis: effect of different renal replacement therapies. Results from the RISCAVID study. Nephrol Dial Transplant 23: 2337 - 2343

11. Grooteman MPC, van den Dorpel MA, Bots ML et al for the CONTRAST Investigators 
(2012). Effect of online hemodiafiltration on all-cause mortality and cardiovascular outcomes. J Am Soc Nephrol 23: 1087 - 1096

12. Ok E, Asci G, Toz H et al on behalf of the "Turkish Online Haemodiafiltration Study" (2013). Mortality and cardiovascular events in online haemodiafiltration (OL-HDF) compared with high-flux dialysis: results from the Turkish OL-HDF Study. Nephrol Dial Transplant 25: $192-202$

13. Maduell F, Moreso F, Pons M et al for the ESHOL Study Group (2013). High-efficiency postdilution online hemodiafiltration reduces all-cause mortality in hemodialysis patients. J Am Soc Nephrol 24: $487-497$

14. Blettner M, Sauerbrei W, Schlehofer B et al (1999). Traditional reviews, meta-analyses and pooled analyses in epidemiology. Int J Epidemiol 28: 1 -9

15. Mostovaya IM, Blankestijn PJ, Bots ML et al on behalf of the EUDIAL Working Group (2014). Clinical evidence on hemodiafiltration: a systematic review and a meta-analysis. Semin Dial 27: $119-127$

16. Nistor I, Palmer SC, Craig JC et al (2014). Convective versus diffusive dialysis therapies for chronic kidney failure: an updated systematic review of randomized controlled trials. Am J Kidney Dis 63: 954 - 967

17. Susantitaphong P, Siribamrungwong M, Jaber BL (2013). Convective therapies versus lowflux haemodialysis for chronic kidney failure: a meta-analysis of randomized controlled trials. Nephrol Dial Transplant 28: 2859 - 2874

18. Wang AY, Ninomiya T, Al-Kahwa A et al (2014). Effect of hemodiafiltration or hemofiltration compared with hemodialysis on mortality and cardiovascular disease in chronic kidney failure: a systematic review and meta-analysis of randomized trials. Am J Kidney Dis 63: $968-978$

19. Tattersall JE, Ward RA; EUDIAL group (2013). Online haemodiafiltration: definition, dose quantification and safety revisited. Nephrol Dial Transplant 28: 542 - 550 
20. Canaud BMM, Jausson I, Cristol JP (2004). Clinical tolerance of online HDF and impact on morbidity and cardiovascular risk factors in ESRD patients of 65 and more years old. Project supported by a French National Grant from Health Ministry (PHRC national)

21. Peters SAE, Bots ML, Canaud B et al on behalf of the HDF Pooling Project Investigators (2016). Haemodiafiltration and mortality in end-stage kidney disease patients: a pooled individual participant data analysis from four randomized controlled trials. Nephrol Dial Transplant 31: $978-984$

22. Grooteman MP, Blanksteijn PJ, Nube MJ (2014). Not all convective dialysis therapies are equal. Am J Kidney Dis 64: 819 - 820

23. Davenport A, Peters SAE, Bots ML et al on behalf of the HDF Pooling Project Investigators (2016). Higher convection volume exchange with online hemodiafiltration is associated with survival advantage for dialysis patients: the effect of adjustment for body size. Kidney Int 89: $193-199$

24. Canaud B, Barbieri C, Marcelli D et al (2015). Optimal convection volume for improving patient outcomes in an international incident dialysis cohort treated with online hemodiafiltration: Kidney Int 88: 1108 - 1116

25. Siriopol D, Canaud B, Stuard S et al (2015). New insights into the effect of haemodiafiltration on mortality: the Romanian experience. Nephrol Dial Transplant 30: 294 301

26. Mostovaya IM, Bots ML, van den Dorpel MA et al (2014). A randomized trial of hemodiafiltration and change in cardiovascular parameters. Clin J Am Soc Nephrol 9: 520 - 526

27. Bellien J, Freguin-Bouilland C, Joannides R et al (2014). High-efficiency on-line haemodiafiltration improves conduit artery endothelial function compared with high-flux haemodialysis in end-stage renal disease patients. Nephrol Dial Transplant 29: 414 - 422

28. Eldehni MT, Odudu A, McIntyre CW (2015). Randomized clinical trial of dialysate cooling and effects on brain white matter. J Am Soc Nephrol 26: 957 - 965 
29. Hsu HJ, Yen CH, Hsu KH et al (2012). Association between cold dialysis and cardiovascular survival in haemodialysis patients. Nephrol Dial Transplant 27: 2457 - 2464

30. Locatelli F, Altieri P, Andrulli S et al (2010). Hemofiltration and hemodiafiltration reduce intradialytic hypotension in ESRD. J Am Soc Nephrol 21: 1798 -1807

31. Cornelis T, van der Sande FM, Eloot S et al (2014). Acute hemodynamic response and uremic toxin removal in conventional and extended hemodialysis and hemodiafiltration: a randomized crossover study. Am J Kidney Dis 64: 247 - 256

32. Daugirdas JT (2016). Lower cardiovascular mortality with high-volume hemodiafiltration: a cool effect? Nephrol Dial Transplant 31: 353 - 356 